\title{
Schwarz Meets Schwann: Design and Fabrication of Biomorphic Tissue Engineering Scaffolds
}

\author{
Srinivasan Rajagopalan and Richard A. Robb \\ Biomedical Engineering Resource, Mayo Clinic College of Medicine, Rochester, USA \\ \{srini, robb.richard\} @mayo.edu
}

\begin{abstract}
Tissue engineering is a discipline at the leading edge of the field of computer assisted intervention. This multidisciplinary engineering science is based on the notion of design and fabrication of scaffolds- porous, threedimensional "trellis-like" biomimetic structures that, on implantation, provide a viable environment to recuperate and regenerate damaged cells. Existing CADbased approaches produce porous labyrinths with contra-naturam straight edges. The biomorphic geometry that mimics the secundam-naturam substrate would be one that is continuous through all space, partitioned into two notnecessarily-equal sub-spaces by a non-intersecting, two-sided surface. Minimal surfaces are ideal to describe such a space. We present results on the premier attempt in computer controlled fabrication and mechanical characterization of Triply Periodic Minimal Surfaces [TPMS]. This initiative is a significant step to link Schwann's 1838 cell theory with Schwarz's discovery of TPMS in 1865 to fabricate the previously elusive optimal biomorphic tissue analogs.
\end{abstract}

\section{Introduction}

Millions of surgical procedures requiring organ (tissue) substitutes to repair or replace malfunctioning organs (tissues) are performed worldwide every year. The everwidening gap between the demand and supply of transplant organs (tissues) has resulted in natural and biomimetic solutions. Autografting, allografting, and tissue engineering are the classical techniques currently pursued to carry out organ transplantation [1]. Tissue engineering involves regenerating damaged or malfunctioning organs from the recipient's own cells. The cells are harvested and embedded on a natural or synthetic carrier material, scaffold, that is ultimately implanted at the desired site. The seeded cells cling, crawl and proliferate within the scaffold. On implantation and integration, blood vessels attach to the new tissue, the scaffold dissolves, and the newly grown tissue eventually blends in with its surroundings. The scaffolds suitable for tissue engineering, apart from being biocompatible and biodegradable, should have highly interconnected pores; have sufficient mechanical properties; and, provide a suitable substrate for cell attachment, migration and proliferation [2].

Tissue engineering scaffolds can be manufactured reproducibly using rapid prototyping approaches like Solid Freeform Fabrication (SFF)- computerized fabrication techniques for rapidly producing complex three-dimensional objects using data generated by CAD systems, medical imaging modalities and digitizers. This 
technique offers unique ways to precisely control substrate architecture for biomimetic structures varying in design and material composition, thereby enhancing control over mechanical properties, biological effects and degradation kinetics of the scaffold. However, the majority of existing approaches under-utilize SFF by producing CADbased scaffolds with straight edges and sharp turns or those derived from Boolean intersections of geometric primitives such as spheres and cylinders. Neither of these partitions provides a biomorphic environment suitable for cell attachment, migration and proliferation [3]. The aggregates of cells, foam/extracellular matrix, typically have cells separated by curved partitions. The biomorphic geometry that best mimics this structural configuration would be surfaces that are continuous through space and divided into two (pore and non-pore) not-necessarily-equal sub-spaces by a nonintersecting two-sided surface. Minimal surfaces are ideal to describe such a space [4]. This paper presents a first practical application of Triply Periodic Minimal Surfaces (TPMS) for the construction of tissue engineering scaffolds. The optimal stress distributions determined from finite element simulations, and mechanical testing, and initial cell viability studies conducted on these scaffolds provide compelling evidence to use them as orthopedic tissue analogs.

\section{Triply Periodic Minimal Surfaces}

A surface $f(x, y, z)=0$ is a minimal surface if and only if the mean curvature $(H)$ at every point of the surface is zero. ie.

$$
H=0=\frac{\left(1+f_{y}^{2}\right) f_{x x}-2 f_{x} f_{y} f_{x y}+\left(1+f_{x}^{2}\right) f_{y y}}{2\left(1+f_{x} f_{x}+f_{y} f_{y}\right)^{3 / 2}}
$$

Any small patch cut from such a surface will have the least area of all the surface patches with the same boundary [5]. TPMS are minimal surfaces periodic in three independent directions, extending infinitely and, in the absence of self-intersections, partitioning the space into two labyrinths. Figure 1 shows the tessellated versions of the TPMS Primitive (P) surface and the Diamond (D) surface discovered by Schwarz [6] and the Gyroid (G) surface discovered by Schoen [7]. The natural manifestation of two, continuous, inter-penetrating, non-intersecting networks in these surfaces has been adapted in natural and man-made environments to concisely describe many seemingly unrelated structures [8]. Though this architecture is a natural fit for the preferred porous and solid subspace topology for tissue engineering scaffolds, to the best of our knowledge, it has not been previously investigated and exploited.

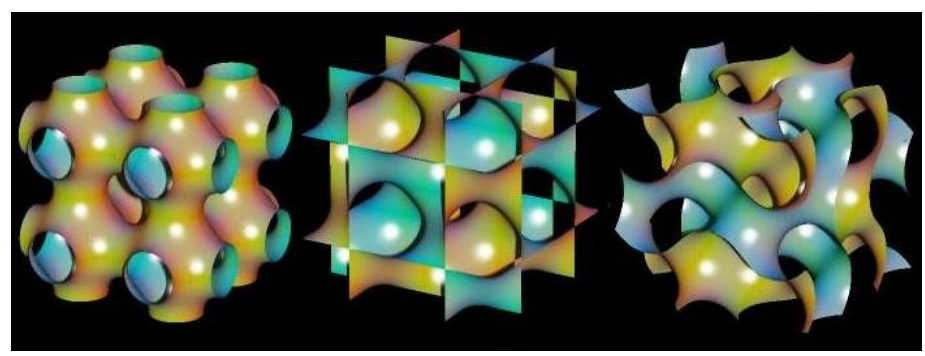

Fig. 1. 3-dimensional tessellations of Schwarz's Primitive (left), and Diamond (centre) and Schoen's Gyroid (right) Triply Periodic Minimal Surfaces 


\section{Geometric Modeling of TPMS}

There are a number of approaches to generate discrete minimal surfaces. Coppin's approach [9] is based on the construction of a quasimolecular model that approximates the molecular structure of a soap film which is a prominent natural manifestation of minimal surfaces. Mackay's construction [10] uses the concept of nodal and Fermi surfaces within a Finite Element formulation. Brakke developed Surface Evolver [11]- a general purpose application that minimizes the energy of a polygonal mesh subject to constraints. Using this approach it is possible to evolve the straight edged, sharply turning cubic labyrinthine network conventionally used in scaffold fabrication into a smooth, curved, non-intersecting, bicontinuous partition. The energy (based on surface tension, gravity and other forms) minimization is performed by evolving the ordered polygonal mesh surface down the energy gradient subject to geometrical constraints on vertex positions and other constraints on integrated quantities such as body volumes. The fully evolved minimal surface can be converted into geometric models that can be realized physically with existing rapid prototyping devices.

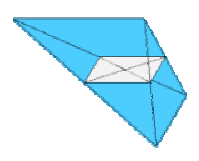

(a)

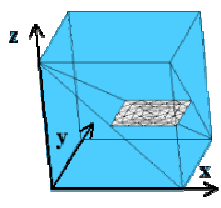

(b)

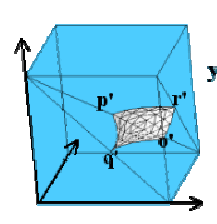

(c)

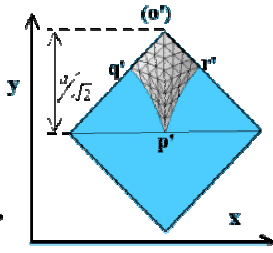

(d)

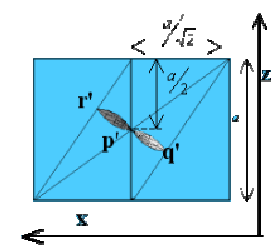

(e)

Fig. 2. Evolution of triangulated plane into a P-surface patch

To generate the fundamental patch of the Schwarz P-surface, quadri-rectangular tetrahedron is used as the fundamental region that confines the initial plane and its subsequent evolved surface. Figure 2 shows the evolution of the plane into a Psurface patch. The plane embedded orthogonally with respect to the faces of the fundamental region (panel a) is refined by subdividing each facet to create a finer triangulation (panel b). The triangulated patch is iteratively evolved to form the Psurface patch (panel c). Panels $d$ and e show the projection of the patch onto the $x y$ and $x z$ planes, respectively. The cube shown in Panels $b$ and $c$ is the bounding cell with edge length $a$.

To form the unit P-cell, the evolved surface patch is reflected around the fundamental region using the line and plane symmetry periodic properties. If part of the boundary of a minimal surface is a straight line, the reflection of the surface across the line, when added to the original surface, forms another minimal surface. If a minimal surface meets a plane at right angles, the mirror image of the surface about the plane, when added to the original surface, also forms a minimal surface. Figure 3 shows the formation of the unit P-cell. Tessellation of the embedded plane (panel a) using the above mentioned properties results in a hollow unit cubic cell (panel b). Tessellation of this cell produces the cubic labyrinthine network (panel c) reminiscent of the existing CAD-based scaffolds. Evolution of (a) leads to the fundamental P- 
patch (panel e) which when replicated using the same tessellation rules produces the unit P-cell (panel f). Tessellation of the unit cells produce the bicontinuous network (panels c,d,g,h). Note that the central hole in both the cubic and P-cell tessellations does not contribute to a pore since the model is not defined therein and hence is treated as belonging to the solid subspace.

Since the P-cell equally partitions the unit cube, the volume should be a half unit; for a continuous P-surface the surface area is 2.3451 [12]. Evolution based on finer triangulation of the initial plane provides better approximation of the discrete surface. However, the complexity of machining increases with the number of polygons. While the volume was preserved across all the triangulations, the optimal balance between polygon count (3072 triangles), surface smoothness and surface area (2.352) was achieved at $2 x$ refinement.

(a)

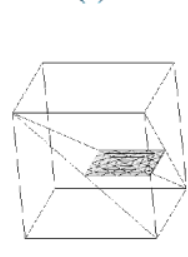

(e)

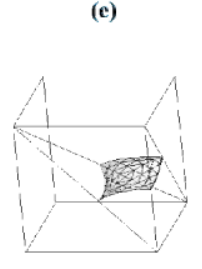

(b)

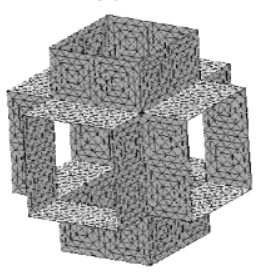

(f)

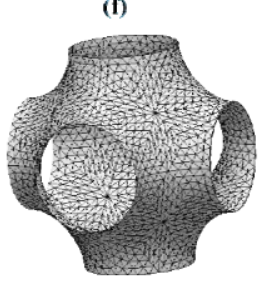

(c)

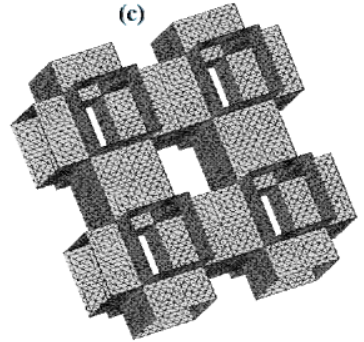

(g)

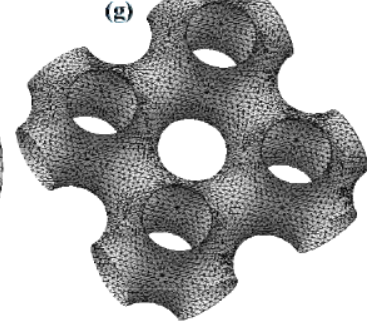

(d)

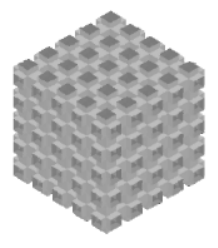

(h)

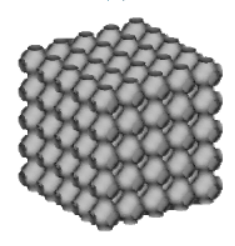

Fig. 3. Using the symmetric periodic properties of minimal surface patches to form the cubic and TPMS unit cells and their tessellations to form scaffolds

\section{Scaled-Truncated TPMS}

The bicontinuous, equi-partitioned triply periodic minimal surfaces have a maximum void area (porosity) of fifty percent. To minimize the amount of material to be washed out on degradation, and to provide sufficient void space for the cells to proliferate, the porosity should be higher. Since a scaled and clipped minimal surface also results in a minimal surface, unit P-cells can be scaled and truncated with a unit cube. The resultant scaled-truncated TPMS (ST-TPMS) would have an increased porosity. Figure 4 shows the effect of scaling the P-and cubic unit cells by 1.25 and truncating the resultant cells by a unit cube. Figure 5 shows the effect of scaling and truncation on the porosity. As is evident from the graph, for the same scale factor, the porosity of the P-cells is higher than that of the cubic cells. At a scaling factor of 1.25, the strut 
width of 200 microns is the minimum feature size that a majority of rapid prototyping devices support. At this level, while the cubic unit cell has a porosity of $68 \%$, the scaled-truncated P-cell has a porosity of $73 \%$. However, the scaling-truncation approach reduces the contact angle between the unit cells. Figure 6 shows the effect of the scaling factor on the contact angle. Though the contact angle decreases, it is still better than the sharp ninety degree turns within the cubic unit cells across all scale factors.

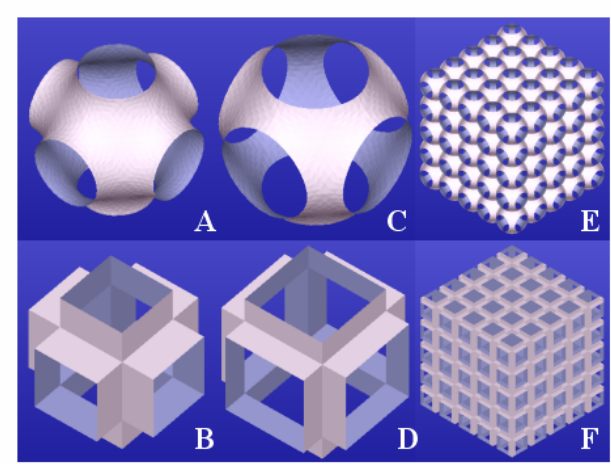

Fig. 4. Scaling and truncation of $\mathrm{P}-(\mathrm{A})$ and cubic (B) unit cells to form unit cells (C, D) and their tessellations $(\mathrm{E}, \mathrm{F})$ with increased porosity. Unit cells in $\mathrm{C}$ and $\mathrm{D}$ are obtained at a scale factor of 1.25

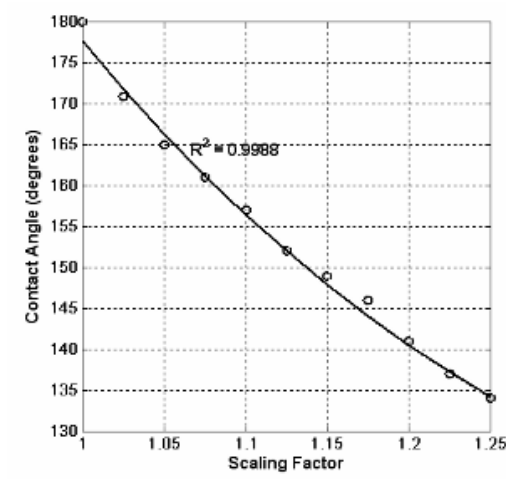

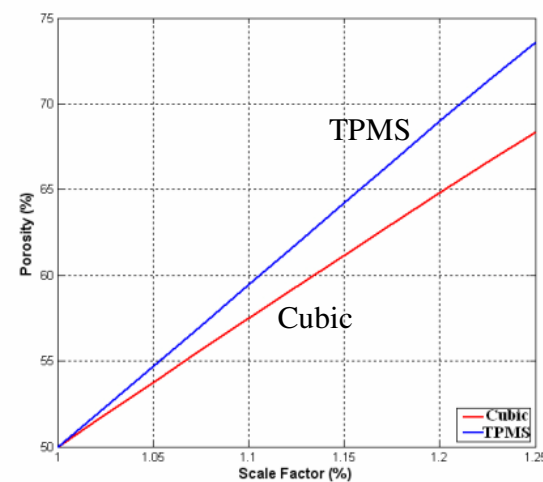

Fig. 5. Effect of scaling and truncation on the porosity of cubic and P- unit cells

Fig. 6. Effect of scaling on the contact angle between the unit P-cells

\section{Fabrication of P-Scaffolds}

The unit cells obtained by evolving and tessellating the plane triangulated at $2 x$ refinement can be directly converted into the .STL stereolithographic format suitable for fabrication with rapid prototyping devices. Multiple copies of the $5 \mathrm{~mm}^{3}$ scaffolds with strut width and pore diameter of 500 microns were fabricated using the Patternmaster rapid prototyping machine (Solidscape, Merrimack, NH) which uses thermoplastic and 
wax to print the solid and pore spaces, respectively. The thermoplastic phase was dissolved in acetone and a biodegradable poly (propylene fumarate) (PPF) polymerizing macromer was injected into the wax mold under a vacuum of $100 \mathrm{mmHg}$. Finally, the wax was melted to form the biodegradable PPF scaffold.

\section{Mechanical Characterization of P-Unit Cells and Scaffolds}

Mechanical Characterization of the unit cells was performed with bulk compressive simulation using ABAQUS (an FEM package). Force of $0.3031 \mathrm{~N}$ was applied to each of the 32 face nodes in the six faces. The material properties (material: PMMA; Young's modulus: $2.4 \mathrm{GPa}$; Poisson ratio: 0.375 ) were set identically for both the cubic and P- unit cells. Figure 7 shows the resultant Von Mises stress and Principal strain distribution for the unit cells. Figure 8 shows the same for the unit cells obtained by scaling the original cells by 1.25 and truncating the resultant with a unit cube. Note that at this scale factor, the porosities of P-cell and cubic unit cells are $73 \%$ and $68 \%$ respectively. It is clearly seen that the stress and strain concentration along sharp edges of the cubic unit cell have been significantly reduced in the unit Pcell. The simulation also reveals the optimal stress distribution and significant strain reduction on the TPMS unit cells.
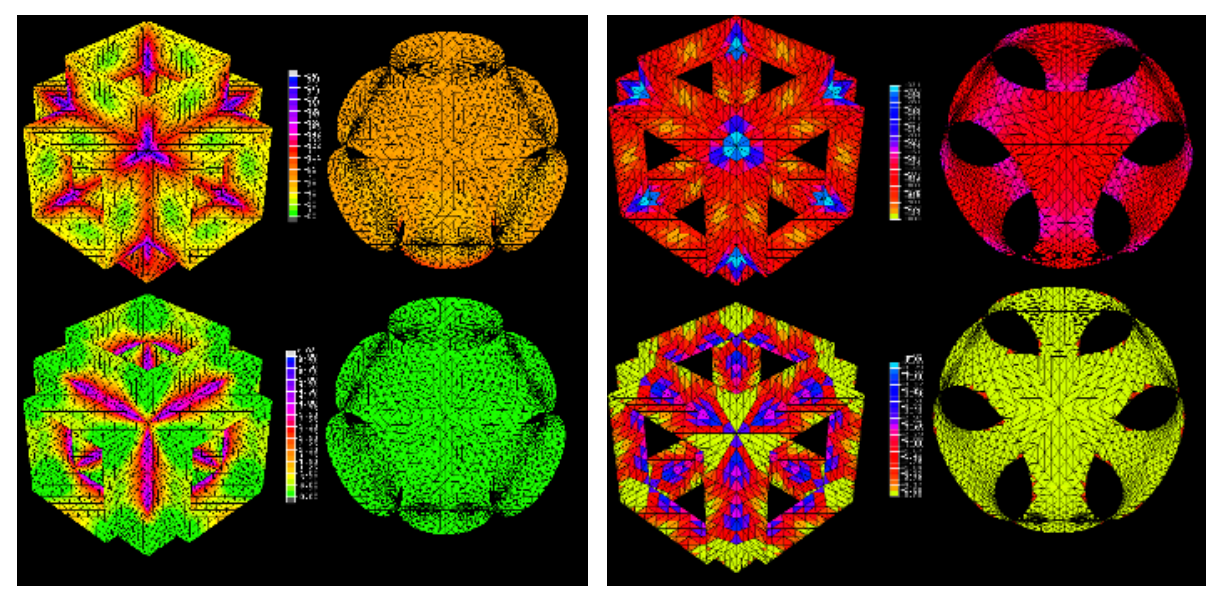

Fig. 7. Von Mises stress (top) and Principal Fig. 8. Von Mises stress (top) and Principal Strain (bottom) maps under bulk Strain (bottom) maps under bulk compression compression for Cubic (left) and TPMS for Cubic (left) and TPMS (right) unit cells (right) unit cells (scale factor 1.0) with (scale factor 1.25) with identical loading identical loading conditions and material conditions and material properties properties

To further validate the simulations and to characterize the whole scaffold, uniaxial compression of the scaffolds and unit cells was performed on a Dynamic Mechanical Analyzer (DMA: TA Inst., New Castle, DE). Fourteen specimens of both the scaffolds were used for the mechanical testing. The specimens were uniaxially 
compressed with parallel plates by applying a ramp force of $4 \mathrm{~N} / \mathrm{min}$ for 4.5 minutes. Figure 9 compares the linear modulus of the cubic and P-cell based unit cells and scaffolds. Figure 10 shows the effect of scaling on the linear modulus of the scaffolds. Both the results correlate with the findings from the finite element simulations.

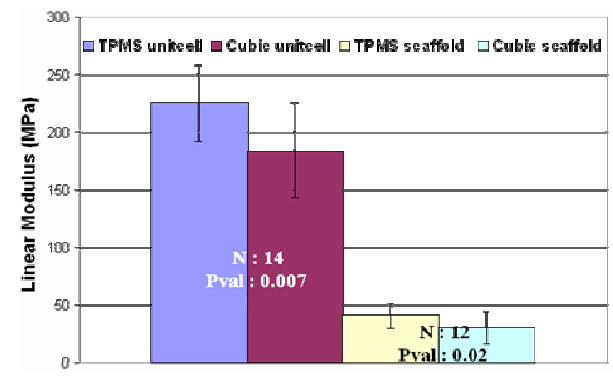

Fig. 9. Linear modulus of TPMS and cubic scaffolds and unit cells based on uniaxial compression on DMA under identical loading conditions (4 N/min. ramp force for 4.5 minutes)

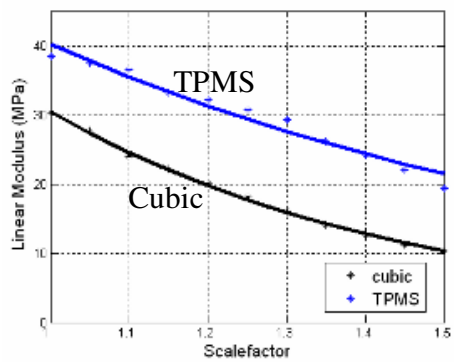

Fig. 10. Effect of unit cell scaling on Linear Modulus based on DMA testing of TPMS and cubic ST scaffolds

\section{Cell Seeding on TPMS Scaffolds}

It is well known that organelles in plant and mammalian cells assume TPMS forms during normal differentiation [13]. To test the suitability of TPMS morphology in tissue engineering, ATDC cells (mouse chondrogenic cell line) were cultured on the scaffolds and examined for viability after 1 day using Viability/ Cytotoxicity kit. Living cells were stained green and dead cells were red when visualized with confocal scanning microscope. A high viability ( $>95 \%)$ was observed for the adherent cells to the surface of scaffolds. Figure 11 shows the distribution of cells on the surface of the scaffold. The cells are active and well distributed through the edges of the pores. The extended cell processes along the scaffold surface reveals the suitability of the substrate.

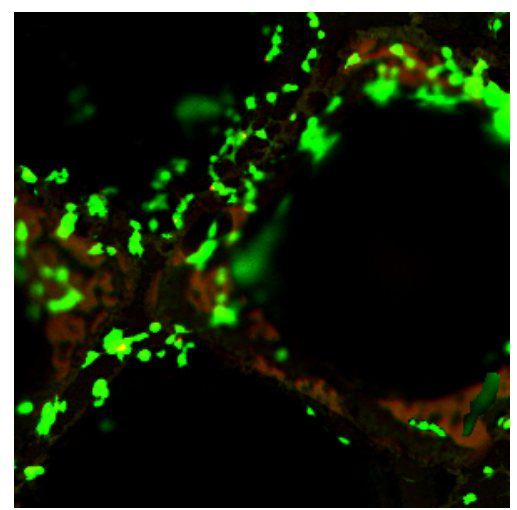

Fig. 11. Suitability of TPMS scaffolds as viable substrate for cell growth demonstrated with confocal scanning microscopy. The live cells are stained in green and the dead cells (representative shown by arrow) in red. As is evident, cell viability is high. 


\section{Conclusions}

Though the natural manifestations of triply periodic minimal surface forms have been observed in living and non-living forms, and the computational framework to create such forms have been in place for decades, to the best of our knowledge, no attempt has been made so far to fabricate them using rapid prototyping devices let alone investigate their potential use as tissue surrogates. This paper describes a first attempt to physically realize and mechanically characterize the Schwarz primitive triply periodic minimal surfaces. The preliminary outcomes of our investigation provide the previously elusive justification for the TPMS-philicity of plant and mammalian organelles. This viable morphology, when replicated at macro (tissue) level may also have profound influence on cell migration and tissue growth and may provide an optimal biomorphic tissue analog.

\section{References}

1. Yaszemski MJ, Payne RJ, Hayes WC, Langer R, and Mikos AG, "Evolution of bone transplantation: molecular, cellular and tissue strategies to engineer human bone", Biomaterials, 17(1996) 175-185.

2. Hutmacher DW, Sittinger M, and Risbud MV, "Scaffold-based tissue engineering: rationale for computer-aided design and solid free-form fabrication systems", Trends in Biotechnology, 22(7) (2004) 354-362.

3. Spalazzi JP, Dionisio KL, Jiang J, and Lu KK, "Osteoblast and Chondrocyte interactions during coculture on scaffolds", IEEE Eng. Med \& Biol., 22(5) (2003) 27-34.

4. Lord EA, and Mackay AL, "Periodic minimal surfaces of cubic symmetry", Current Science, 85(3) (2003) 346-362.

5. Meusnier JBMC, Mem. Mathem. Phys. Acad. Sci. Paris, pres. par. div. Savans, 10 (1785) 477-510.

6. Schwarz H, Gesammelte Mathematische Abhandlungen, Vols 1, 2 (1890), Springer, Berlin.

7. Schoen AH, "Infinite periodic minimal surfaces without self-intersections" NASA Technical report TN D-5541, (1970), Washington DC.

8. Hyde S, Andersson S, Larsson Z, Blum T, Landh S, Lidin BW, and Ninham BW, The Language of Shape. The Role of curvature in Condensed Matter: Physics, Chemistry and Biology, (1997), Elsevier, Amsterdam.

9. Pinkall U, and Polthier K, "Computing Discrete Minimal Surfaces and their conjugates", Experimental Mathematics, 2 (1993) 15-36.

10. Mackay AL, "Periodic minimal surfaces from finite element methods", Chem. Phys. Lett. 221 (1994) 317-321.

11. Brakke KA, "The Surface Evolver", Experimental Mathematics, 1(2) (1992) 141-165.

12. Mackay AL, "Periodic minimal surfaces", Nature, 314 (1985) 604-606.

13. Landh T. FEBS Lett, 369:13-13, 1995. 